\title{
The synergistic anti-hypertensive effects of consuming olive leaf extract alongside increasing physical activity
}

\author{
B. Saibandith ${ }^{1}$, K. Tolfrey ${ }^{2}$, J. Spencer $^{1}$, I. Rowland ${ }^{1}$ and D. Commane ${ }^{1}$ \\ ${ }^{1}$ Hugh Sinclair Unit of Human Nutrition, Department of Nutritional Sciences, University of Reading, Earley Gate, \\ Whiteknights, Reading, UK, RG6 6AL and ${ }^{2}$ School of Sport, Exercise and Health Sciences, Loughborough University, \\ Epinal Way, Loughborough, Leicestershire, UK, LE11 $3 T U$
}

Public health guidelines advocate a low sodium intake, a healthy diet and a physically active lifestyle for the prevention of hypertension. Supplementation with anti-hypertensive functional foods may also be desirable. The olive leaf is a waste product of olive production and is rich in polyphenols which can be recovered in extracts (OLE) for supplementation. Recent randomised control trials demonstrate hypotensive ${ }^{(1)}$ and anti-glycaemic activity ${ }^{(2)}$ for OLE in human volunteers.

Advocating the consumption of a supplement for the prevention of hypertension is a sub-optimal public health approach as supplement usage can be used to justify an otherwise unhealthy lifestyle. Accordingly, evidencing the synergistic benefits of including a supplement as part of a healthy lifestyle is desirable. This study therefore aims to evidence the synergistic benefits of consuming plant (and specifically olive) phenolics alongside being more physically active in individuals with elevated blood pressure.

In this randomized, four-arm parallel study, 112 pre-hypertensive overweight adults aged 25-70y are assigned to one of four treatment arms: i) a placebo control group- with a daily capsule for 12 weeks; ii) an increasing physical activity and a placebo capsule group for 12 weeks; iii) an olive leaf extract arm (equivalent to $132 \mathrm{mg}$ of oleuropein) for 12 weeks; iv) a physical activity and olive leaf extract arm. The study is double blinded to OLE vs placebo. Individuals on the physical activity arms are equipped with pedometers as motivational tools, they also receive weekly postal guidance on gradually increasing physical activity over 12 weeks, and are offered support to do this through social media.

The primary endpoint is the change in 24 hour ambulatory blood pressure. Secondary outcome measures for the study are digital pulse wave velocity (as a measure of vascular function), plasma HbA1C levels (a measure of chronic blood sugar control), serum lipid profile and body composition. Adherence to the treatment arms is assessed via the analysis of plasma for metabolites of olive extract and through accelerometer data.

Preliminary analysis demonstrates that the physical activity intervention successfully induces behaviour change over the 12 weeks. Higher physical activity and a decrease in sedentary behaviour is associated with a reduction in blood pressure. The OLE/placebo data are as yet un-blinded but preliminary analysis shows a separation of study arms.

This holistic approach to the study of nutrition and physical activity synergistically, through intervention study, may better evidence current public health guidelines.

1. Lockyer S, Rowland I, Spencer JP, et al. (2016) Eur J Nutr. [Epub ahead of print]

2. De Bock M, Derraik JG, Brennan CM et al. (2013) PLoS One 8, 1-8 